\title{
4BeGreen: Metodología para motivar la conciencia ambiental, la valorización y prevención de residuos en empresas de servicios
}

\author{
4BeGreen: A methodology to encourage environmental awareness for recovery, and \\ prevention of waste in service companies
}

Luis Eduardo Cañón-Ramirez ${ }^{1}$, Vanessa Prieto-Sandoval ${ }^{2}$

Recibido: Agosto 2020

Aceptado: Diciembre 2020

Resumen.- La operación de una organización de servicios puede crear el imaginario de una reducida generación de impactos ambientales negativos, esto se evidencia en la ausencia de estrategias de gestión ambiental al interior de la empresa tanto en su operación como en los hábitos de sus empleados. Sin embargo, este es un tema que cobra cada vez más importancia en las organizaciones por los beneficios para la empresa y sus empleados. La empresa se beneficia en términos de recordación de marca, prestigio, beneficios económicos y legales. De la misma manera, los colaboradores pueden sentir mayor sentido de pertenencia, lealtad hacia la empresa e incrementar su sentido de retribución al sentir que actúan de manera ética. Con el objetivo de proponer una metodología para motivar la conciencia ambiental, la valorización y prevención de residuos en empresas de servicios, este estudio desarrolló un caso de estudio en una empresa de servicios de exportación y negociación internacional. Como resultado se diseñó la metodología 4BeGreen, compuesta por 4 pasos concretos: 1) Diagnóstico, 2) Caracterización de residuos, 3) Plan de concientización teórica y práctica, 4) Evaluación ecológica y retroalimentación.

Palabras clave: Valorización de residuos, Economía circular, sostenibilidad, gestión ambiental, empresas de servicios.

Summary.- The operation of a service organization can create an image of a reduced generation of adverse environmental impacts. It is evidenced by the absence of environmental management strategies within the company both in its operation and in the habits of its employees. However, it is an increasingly important topic in organizations based on the benefits for the company and its employees - the company benefits in terms of brand awareness, prestige, economic benefits, and legal interests. In the same way, employees can feel a greater sense of belonging, loyalty to the company, and increase their sense of retribution by feeling that they are ethical. Intending to propose a methodology to motivate environmental awareness, recovery, and prevention of waste in service companies, this study developed a case study in an export service company. As a result, the 4BeGreen methodology was designed, consisting of 4 concrete steps, representing benefits for the company and its collaborators. 1) Diagnosis, 2) Waste characterization, 3) Theoretical and practical awareness plan, 4) Ecological evaluation and feedback.

Keywords: Waste recovery, circular economy, sustainability, environmental management, service companies.

\footnotetext{
1 Depto. de Sostenibilidad, Procolombia, lcanon@procolombia.co, ORCID iD: 0000-0002-9852-1554

2 Depto. de Administración, Pontificia Universidad Javeriana, juliethv.prieto@javeriana.edu.co, ORCID iD: 0000-0001-9937-7710
} 
Introducción.- El desarrollo sostenible es un compromiso global, que ha cobrado especial importancia desde el año 2015, con la firma del COP21 en París donde se estableció el compromiso de limitar el aumento de la temperatura media global por debajo de $\operatorname{los} 2^{\circ} \mathrm{C}$, la publicación del Laudato Sí, y el mandato otorgado por las naciones unidas al Pacto Global para sensibilizar a las organizaciones privadas y comprometerles para contribuir al cumplimiento de la nueva agenda para el desarrollo establecida en los Objetivos de Desarrollo Sostenible a 2030 [1]. En el marco de los desafíos ambientales y sociales de esta época, la Economía Circular (EC) se presenta como un modelo económico alternativo que facilita el desarrollo sostenible, impulsa la innovación para el cierre de flujos de materiales y energía, y tiene un alcance multinivel, donde países, agrupaciones industriales y empresas pueden generar estrategias para su implementación [2].

La implementación en las empresas usualmente se relaciona con prácticas de producción más limpia para mejorar los procesos y prácticas de la empresa [3], las estrategias de diseño sostenible para bienes y servicios [4-6], logística inversa que facilita la recuperación de los bienes después de su uso para seleccionar la opción de disposición final más efectiva o recuperación de los materiales a través de modelos de negocio relacionados con la remanufactura, restauración, reutilización o reciclaje [7-8], programas de capacitación ambiental y técnica para la fuerza laboral [9-10].

En este sentido, desde el sector privado, las empresas están llamadas a generar estrategias que permitan concienciar a sus empleados sobre la importancia de la sostenibilidad ambiental y los beneficios que puede traer para la prosperidad social y económica del negocio. Son varias las metodologías para mejorar el conocimiento, la conciencia y la preocupación por el ambiente en las empresas. Según Ormazabal et al. [11] las certificaciones de gestión ambiental son útiles para mostrar la estandarización de la gestión ambiental en una organización con madurez intermedia. La razón es que las normas proporcionan especificaciones y directrices nacionales e internacionales para los sistemas de gestión ambiental [12]. En este sentido, los productos y servicios de las empresas también pueden certificarse, un ejemplo es el ecoetiquetado, que es la manifestación visible de un proceso voluntario de eco-innovación. En este proceso, se cumplen las expectativas ambientales de los consumidores, las empresas aumentan su valor creado y capturado y mejoran su sostenibilidad, y los gobiernos e instituciones fomentan la producción y el consumo más limpios [13]. Las etiquetas ecológicas simplifican el proceso de toma de decisiones de los consumidores, y esto indica que están eligiendo un bien o servicio "verde" [14].

Por otra parte, la gestión ambiental de las empresas también requiere la caracterización y apropiada gestión de residuos que pueden ser generados en el proceso productivo o en el posconsumo. Según Smith, R. [15] la gestión adecuada de los residuos sólidos y el reciclaje disminuye la cantidad de materia prima virgen usada en procesos productivos de bienes y servicios, ya que, al ingresar de nuevo los materiales recuperados a las cadenas de valor, son transformados para darle vida a otros productos. "Hacia 1990 países como Dinamarca y Alemania intensificaron los programas de reciclaje, logrando reducir hasta un $90 \%$ de materia prima virgen", lo que evidencia que una gestión adecuada de residuos no solamente disminuye el impacto ambiental negativo generado en los vertederos, sino que se convierte a su vez en una oportunidad de generación de ingreso y disminución en costos de producción para las organizaciones.

Sin embargo, se evidencia un vacío en la literatura relacionado con la ausencia de una metodología que cumpla con la función de crear conciencia y conocimiento en los empleados; y a la vez mejorar la gestión ambiental reduciendo y valorizando los residuos de las empresas, y así contribuir con a la implementación de la economía circular. Una búsqueda usando la ecuación TEMA: ("circular economy") AND TEMA: (employee*) en la base de datos Web of Science hecha en Julio de 2020, mostró únicamente 36 resultados. Luego la ecuación TEMA: ("environmental management") AND 
TEMA: (employee*) mostró 461 publicaciones, pero solo 55 pertenecen a Latinoamérica (ninguna en Colombia), y más del $80 \%$ fueron publicadas en los últimos 10 años. Una tercera ecuación de búsqueda con "Trainning" AND "Environmental awareness", generó 835 resultados, de los cuales solo 50 son de Latinoamérica y 111 son del año 2020. Por otra parte, este artículo se centra en las empresas de servicios ya que estas presentan un especial desafío porque sus operaciones no generan residuos directamente relacionados con actividades manufactureras, sino que están relacionados con actividades de backoffice, así como los hábitos de consumo de quienes allí trabajan. Es importante resaltar que gran parte de los procesos, modelos o metodologías de gestión ambiental se enfocan en empresas de manufactura debido a la generación directa de un alto volumen de residuos, y no atienden las oportunidades de implementación de la economía circular en empresas de servicios.

Por tanto, el objetivo principal de este artículo es diseñar una metodología para motivar la conciencia ambiental, la valorización y prevención de residuos en empresas de servicios. Luego como segundo objetivo, este estudio valida la efectividad de la metodología con un caso demostrativo, mostrando los sus beneficios, ambientales, sociales y económicos.

Después de esta introducción, la sección 1 presenta los fundamentos teóricos, la sección 2 explica la metodología utilizada en este estudio. En la sección 3 se presenta la propuesta metodológica, los resultados del caso de estudio y su contribución. Finalmente, en la sección 4 se presentan las conclusiones y se identifican las áreas de investigación futura.

\section{Revisión de la literatura.-}

2.1. Implementar la Economía Circular con los empleados.- La gestión ambiental en las organizaciones "implica el estudio de todas las actividades técnicas y organizativas destinadas a reducir el impacto ambiental causado por las operaciones comerciales de una empresa" [16]. Pero la gestión ambiental de las empresas se encuentra en diferentes etapas de madurez [11]. Significa que el comportamiento de las empresas y la forma en que enfrentan los desafíos ambientales están más o menos avanzadas en cada empresa [17].

En la misma línea de actuación, La ONU HABITAT [18] se ha encargado de promover la iniciativa global denominada Waste Wise Cities, cuyo objetivo es promover ciudades inteligentes en el manejo de los residuos a través de cinco acciones básicas, "Repensar, Rechazar, Reducir, Reutilizar y Reciclar". Esta campaña se convierte en una gran oportunidad para que las organizaciones promuevan practicas encaminadas a la implementación de las cinco acciones básicas mencionadas anteriormente y que a su vez contribuyen al cumplimiento de los ODS orientados a mitigar los impactos ambientales negativos que genere la operación de una organización

Aun así, el alto grado de madurez de la gestión y el conocimiento ambientales de una compañía, no siempre garantizan que este conocimiento sea compartido por todos los empleados. Considerando que las empresas son compuestas por personas con ideas, cultura y motivaciones[1921], la gestión del talento humano es uno de los temas más relevantes para la implementación de la EC [22]. La teoría y la práctica indican que todos los empleados deben ser considerados como gerentes ambientales y empoderarlos con la posibilidad de mejorar el desempeño de las empresas en este aspecto [23], a través de conocimiento ambiental, conciencia y preocupación para implementar prácticas ecológicas [24].

Sin embargo, no todas las compañías están desarrollando programa para crear conciencia y el compromiso de los empleados para promover prácticas empresariales en el marco de la economía 
circular y de "cero residuo[22]. Incluso, empresas como Biogen, con estrategias de "cero residuos" han identificado que una conciencia limitada sobre la separación y eliminación de los desechos de la cocina y la oficina. $43 \%$ de los empleados encuestados informaron que la señalización para separar los desechos del reciclaje y el compostaje no era clara, resultado que tenía impacto directo en la eliminación inadecuada de materiales reciclables en contenedores de desechos sólidos (en particular, cartón y utensilios de cocina compostables).

Así que, tal como lo explican Veleva et al. [22] se requieren metodologías y herramientas que para involucrar a los empleados en la implementación de la economía circular en las empresas, así como la medición eficaz de la sensibilización y el avance de la reducción, reutilización y remanufactura de bienes. Por lo cual, Veleva et al. [22] propone la promoción de "cero residuo" y Economía Circular, con el modelo "Expanded zero waste practice" donde proponen usar la jerarquía de reducción de desechos de reducir-reutilizar-reciclar y compostar-recuperación energéticadisposición final, y a la vez incorpora dos dimensiones. Primero, el diseño de indicadores para medir los "resultados" e "impactos" de cada estrategia de reducción de residuos y en segundo lugar el "desperdicio cero" como parte integral de una estrategia de participación de los empleados en la que los empleados están informados, educados y capacitados para tomar medidas.

2.2. Empresas de servicios.- Según la Organización Mundial del Comercio el sector de los servicios representa el mayor porcentaje de la economía mundial (65\%) y genera el $49 \%$ de los empleos, en Latinoamérica y el caribe tiene un peso del $61 \%$ en el PIB y genera el $65 \%$ de los empleos aproximadamente [25]. Además. el comercio de servicios tiene un gran potencial para contribuir a la optimización de recursos, generar economías de escala, diversificar la oferta, e impulsar la productividad[25]. En este sentido, la innovación y el desarrollo de mejores prácticas de gestión ambiental puede tener un impacto en más de la mitad de las empresas y trabajadores en el mundo.

Sin embargo, solo en España el sector servicios genera un total de 5.698.000 toneladas de residuos al año y el 60\% de estos provienen del comercio, así mimos el 34,5\% de los plásticos que se generan en España provienen del sector servicios[26]. Así, se puede decir que las empresas de servicios tienen la responsabilidad de implementar estrategias que permitan mitigar su impacto ambiental. Para ello pueden implementar técnicas de reciclaje, entendido como "aprovechamiento total o parcial de los residuos industriales para su reutilización en el propio proceso".[27] Ya que, sin importar su objeto social estas generan residuos que deben ser tratados adecuadamente para impedir su llegada a los vertederos.

3. Metodología.- Para este estudio se hizo una revisión de literatura para el desarrollo del estado del arte y diseño de la metodología. Luego, se desarrolló un caso de estudio para validar su efectividad en una empresa de servicios de exportación y lobby de comercio internacional que para este estudio se llamará CoExport.

La revisión de literatura se enfocó en identificar modelos y estrategias para implementar la Economía Circular con ayuda de los empleados en literatura académica y literatura gris. Las ecuaciones de búsqueda que se utilizadas fueron TEMA: ("Economía Circular") AND TEMA: (empresas), TEMA: ("circular economy") AND TEMA: (employee engagement), así como TEMA: ("economía circular") AND TEMA: (sector servicios) en Julio de 2020, en la base de la Pontificia Universidad Javeriana que incluye colecciones como Fuente Académica Premier, Complementary Index, eBook Collection (EBSCOhost), eBook Academic Collection, entre otras.

Los casos de estudio exploran y estudian fenómenos de la vida real a través del análisis contextual

Memoria Investigaciones en Ingeniería, núm. 19 (2020). pp 63-75

https://doi.org/10.36561/ING.19.5

SSN 2301-1092 • ISSN (en línea) 2301-1106 
detallado de un número limitado de eventos o condiciones y sus relaciones [28].En este sentido, el caso de estudio se centró en la importancia de transformar las políticas de gestión de residuos de los países y las ciudades en acciones concretas que permita generar alternativas sostenibles [29] en las empresas de servicios y de esta manera definir planes de educación ambiental que impacten a las comunidades en general. Es por esta razón que, este estudio plantea una serie de pasos para que se concreten acciones reales que promuevan hábitos sostenibles en los colaboradores de esta organización que se reflejen en su trabajo con resultados medibles.

3.1. Contexto.- Este estudio se desarrolló en la empresa que para el estudio fue nombrada CoExport, fundada en 1992 en Colombia, con 490 de empleados, y presente en 9 departamentos y 25 países. La empresa contaba con un modelo de sostenibilidad integral, en el cual se definían parámetros y estrategias encaminadas a la sostenibilidad ambiental en la gestión interna. Este modelo se alinea con la evolución del país, ya que Colombia ha iniciado su transición hacia la economía circular tras casi tres décadas de reestructuración legal en el sector de residuos[30].

3.2. Desarrollo del caso.- El caso se llevó a cabo del 13/08/2019 a 30/03/2020 en las ofícinas de Bogotá, Colombia, con la participación de 200 empleados, ubicados en 4 pisos de un edificio de más de 40 pisos. De acuerdo con Sanchez-Muñoz et al. [31], cerca del $80 \%$ de la población bogotana reconoce en gran medida la importancia del reciclaje, sin embargo, muy pocos no conocen los conceptos básicos, los procesos de separación adecuadas y los objetivos de la separación de residuos. Por lo que se hace necesaria que las organizaciones como CO-EXPORT implementen iniciativas que promuevan la gestión adecuada de residuos sólidos, que a su vez promuevan el reciclaje, la reutilización, la reducción, el rechazo y el repensar los hábitos de consumo.

Con el diseño e implementación de la metodología se espera que haya un aumento del material reciclado (cantidad mensual de material entregado al operador de reciclaje), aumento en la participación de los colaboradores (fotografías, listas de asistencias a las actividades, número de participantes), disminución en el consumo del agua (medición recibos públicos), y disminución en el uso de plásticos de uno solo uso y polietileno expandido.

4. Resultados y discusión.- En esta sección se presenta la propuesta metodológica de 4BeGreen, explicando cada uno de los pasos clave.

4.1. Propuesta metodológica 4BeGreen.- Con base en la revisión de literatura se identificó que la metodología del Jaca et al. [32] y el programa , La ONU HABITAT [18] proporcionaba un paso a paso y estrategias clave para incrementar la conciencia y el comportamiento sostenible de los empleados, respectivamente. Jaca et al. [32] plantea la importancia de desarrollar elementos de diagnóstico, un plan de acción, ejecución y evaluación para implementar la economía circular en las empresas. Así se diseñó la metodología 4BeGreen, con cuatro pasos clave: 1) Diagnóstico, 2) Caracterización de residuos, 3) Plan de concientización teórica y práctica, 4) Evaluación ecológica y retroalimentación ilustrada en la figura I. 


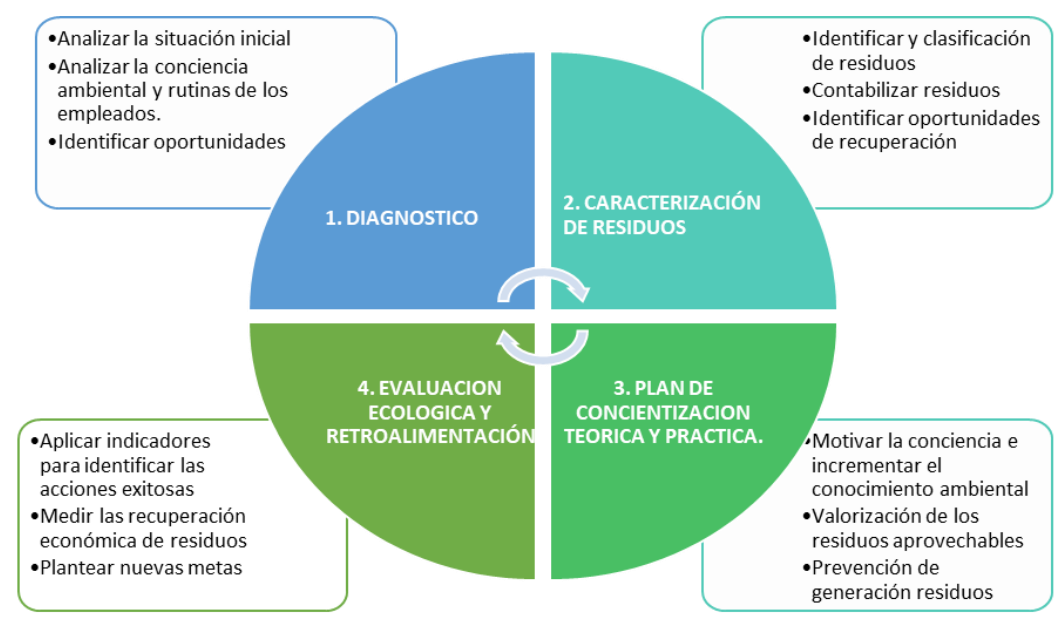

Figura I.- Metodología 4BeGreen.

4.2. Diagnóstico.- En esta primera fase, el diagnóstico tiene el propósito de analizar la situación actual de las prácticas ambientales de la organización, así como la conciencia ambiental y rutinas de los empleados. Para este propósito se aplicó la herramienta "Journey map" presentado en la figura II. Un “journey map” es una herramienta de clarificación, para comprender la experiencia de los usuarios a través de la identificación cronológica de las actividades clave que realizan los usuarios. Se hace revisando la lista completa de actividades desarrolladas por los usuarios o en este caso el "staff", luego se realiza un análisis de cluster de las principales actividades, las cuales se pueden mostrar como nodos en una línea de tiempo[33]. A través de recorridos de observación que permitieron identificar las condiciones de los puntos ecológicos, la separación de residuos y el comportamiento de los colaborares durante la separación en los puntos ubicados en los cuatro pisos de la organización. Dichos recorridos se realizaron de la siguiente manera: el primer día se realizó el recorrido antes del mediodía del martes, el según día se realizó después de las 14 horas del miércoles. Esta observación se realizó de forma indirecta ya que los individuos no sabían que su actuar estaba siendo observado, estos comportamientos fueron consignados en una hoja de registro diseñada para tal fin. Después de eso, se identifican acciones positivas y negativas. Finalmente, la tarea más importante es identificar conocimiento nuevo acerca de los desafíos y oportunidades de innovación en la rutina analizada o un proceso. 


\section{Journey Map empleados CoExport}

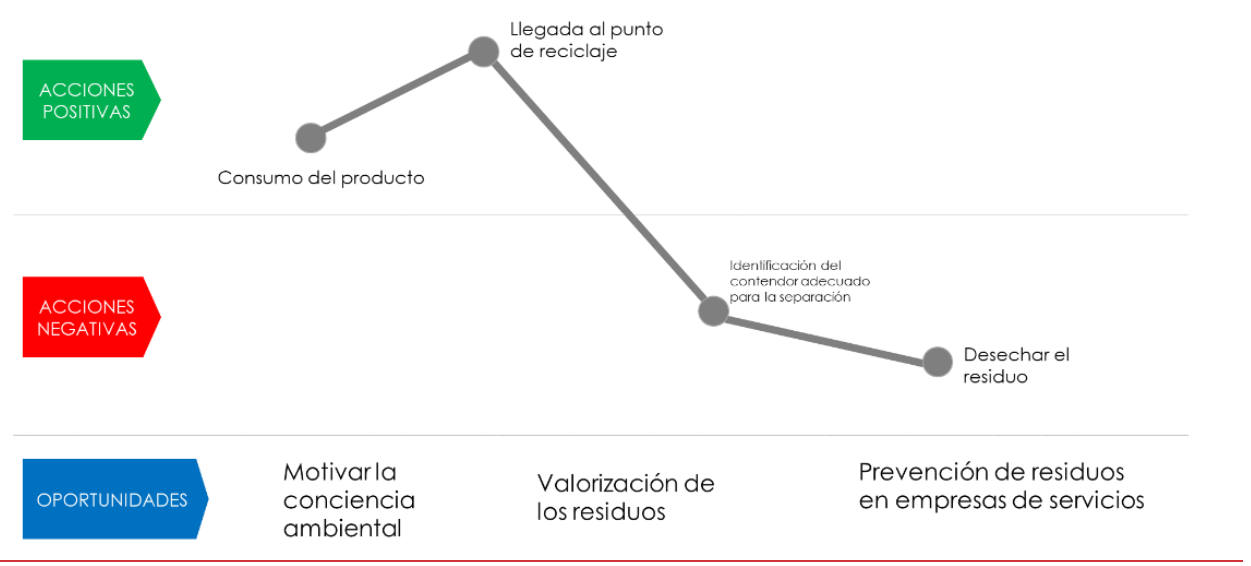

Figura II.- Journey Map empleados CoExport.

Estos recorridos permitieron dar cuenta de la deficiencia general de las prácticas ambientales y sobre todo de la falta de información y orientación frente a la disposición final de los residuos ya que se evidenciaron rutinas en el comportamiento de los colabores, en cuatro elementos: personales, contextuales, sociales y organizacionales. Primero, en cuanto a los elementos personales, son los que tienden a requerirse con inmediatez en rutinas rápidas de uso, y hay alto desconocimiento del impacto a futuro. Segundo, los elementos contextuales, durante la observación se evidenció que los empleados prestaban poca atención a los colores de demarcación de las canecas de disposición de residuos y el comportamiento es mimético, así que las personas tienen a usar la caneca que usan sus compañeros sin criterios ambientales. Luego, los elementos sociales se refieren a la baja conciencia ambiental que es ocasionada por la falta de sanciones y divulgación adecuada de programas distritales o nacionales de reciclaje y/o separación de residuos y economía circular. Finalmente, los elementos organizacionales consisten en las normas ambientales de la empresa, pero no había una "sanción organizacional" por el uso inadecuado de las canecas ni incentivos apropiados para motivar la apropiada separación de residuos.

4.3. Caracterización de residuos.- En esta segunda fase, durante 4 días (lunes a jueves) se llevó a cabo la caracterización de los residuos mediante el formato de "Caracterización de residuos sólidos" (Figura III) de CoExport que establece el tipo de residuos, cantidad y peso, con el objetivo de identificar los tipos de residuos que se estaban generando. Para lo anterior, se realizó un proceso de clasificación, conteo y pesaje de los residuos depositados en los 8 puntos ecológicos de la organización, ubicados en los 4 pisos ( 2 en cada piso) entre las 14 y las 16 horas, para tener los residuos acumulados en el día.

Como resultados de esta caracterización se obtuvieron $29.5 \mathrm{~kg}$ de residuos orgánicos, $2.5 \mathrm{~kg}$ de residuos de papel, otros residuos aprovechables como 21 botellas de vidrio, 20 latas de aluminio, 7 cajas Tetrapak, 114 botellas PET. El resto eran materiales no aprovechables contaminados con alimentos (Figura IV). 


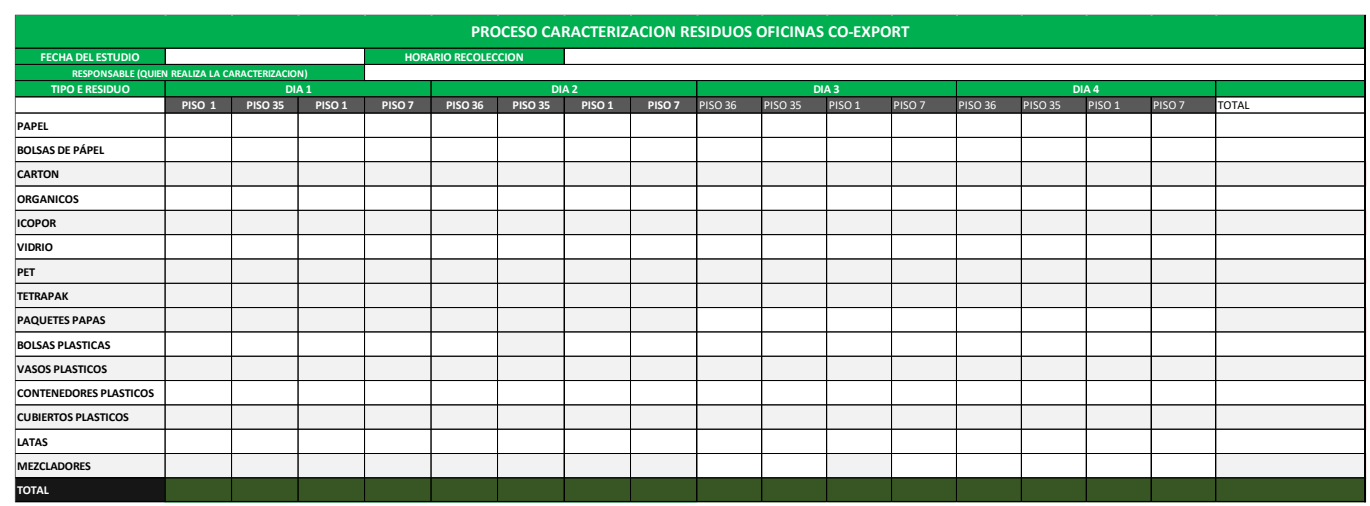

Figura III.- Caracterización de residuos sólidos 4BeGreen.
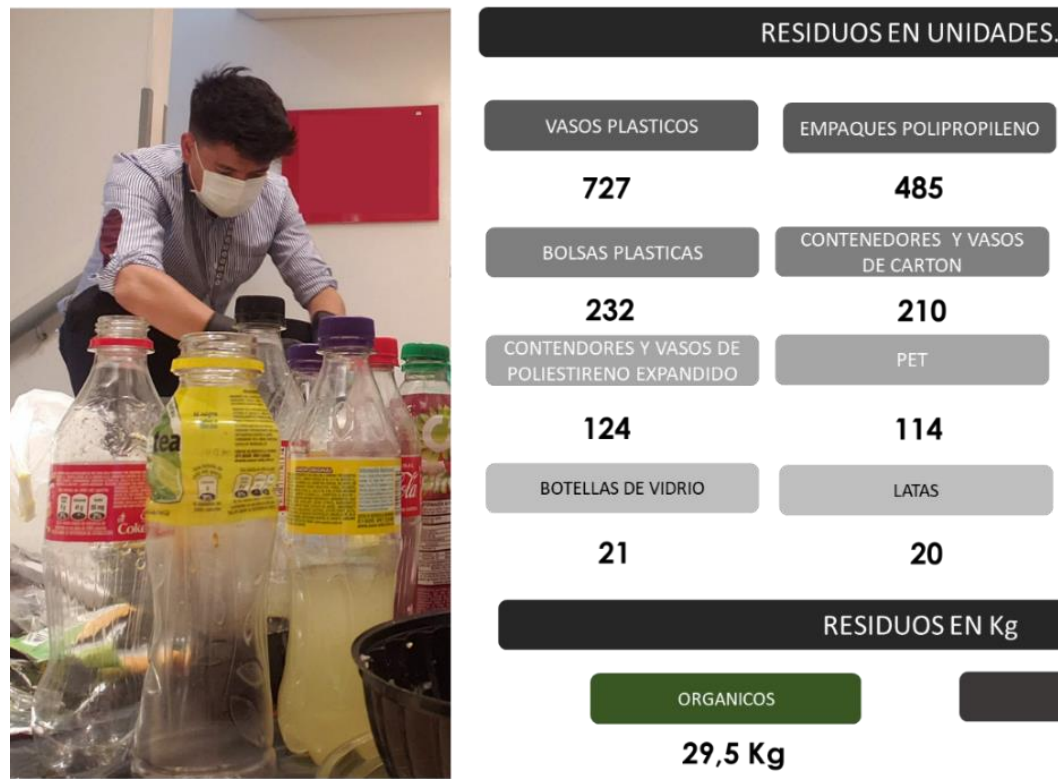

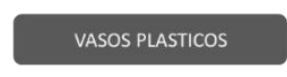

727

BOLSAS PLASTICAS

232

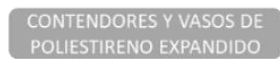

124

BOTELLAS DE VIDRIO

21
EMPAQUES POLIPROPILENO

485

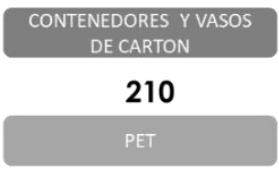

114

LATAS

20
MEZCLADORES Y PITLLOS

352

CUBIERTOS PLASTICOS

156

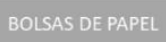

91

TETRAPAK

7

\section{RESIDUOSEN Kg}

ORGANICOS

$29,5 \mathrm{Kg}$
PAPEL

$2,5 \mathrm{Kg}$

Figura IV.- Caracterización de residuos, hecha entre el 13 y 16 de agosto de 2019.

4.4. Plan de concientización teórica y práctica.- Una vez identificados los residuos generados, los hábitos de consumo y posconsumo, se estableció un plan de concientización teórico-práctico que permitiría generar estrategias para concienciar a los colaboradores sobre la importancia de la sostenibilidad ambiental y los beneficios que pueden traer. Así mismo, la concientización tiene el propósito de fortalecer las practicas del BackOffice, bajo una campaña sombrilla que reuniría todas las actividades propuestas, para efectos de este articulo la campaña de llamará VIVIENDO EL AMBIENTE.

En esta fase de establecieron actividades dirigidas a tres propósitos; 1) motivar la conciencia e incrementar el conocimiento ambiental, 2) la valorización de los residuos aprovechables y 3) prevención de generación residuos, como lo muestra la figura V. Primero, la conciencia y el conocimiento se incrementaron con concurso que premia el comportamiento, hábitos y consumo responsable, así como la adecuada separación de residuos. En este sentido, también se promovieron 
jornadas de voluntariado para siembra de árboles, limpieza y recuperación de parques y zonas verdes. En segundo lugar, la valorización de residuos se desarrolló a través de un programa externo de economía circular que transforma residuos plásticos en madera plástica. También se coordinó y ejecutó la venta de material reciclado a gestor ambiental, el dinero obtenido se reinvirtió en actividades con comunidades vulnerables o en riesgo de exclusión. Finalmente, la prevención de generación de residuos se ha logrado con jornadas de sensibilización con operadores de reciclaje y recuperadores ambientales para entender la adecuada separación de residuos en la fuente. Además, se han diseñado programas para la sensibilización frente al consumo y eliminación de plástico de un solo uso.
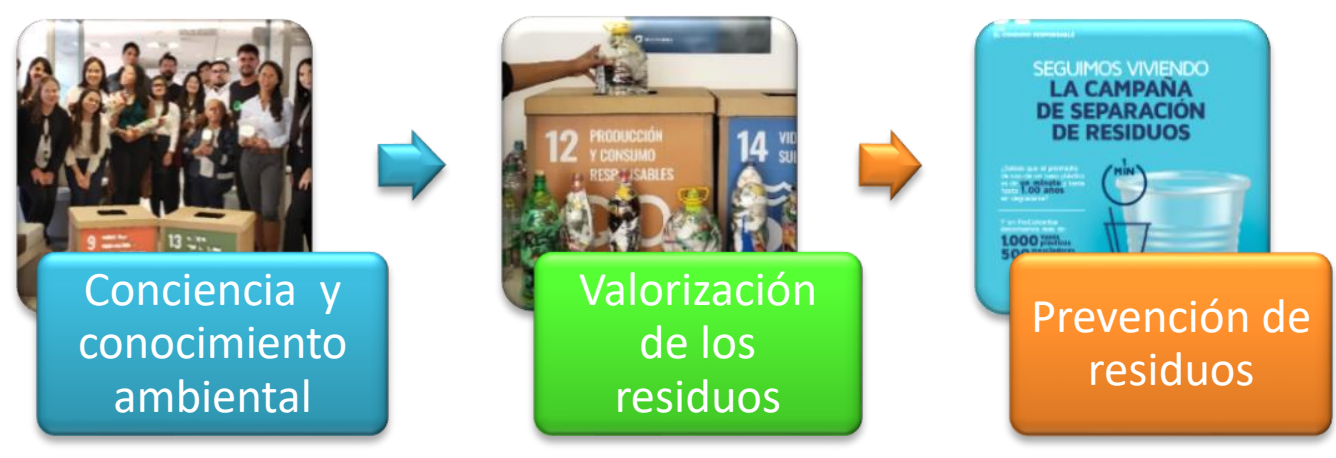

Figura V.- Concientización ambiental teórico-práctica.

4.5. Evaluación ecológica y económica de resultados.- Durante la implementación de la estrategia se han logrado avances significativos en las prácticas de gestión ambiental, que además han contribuido a la implementación de la economía circular al interior de la organización.

Frente al impacto ecológico, ha aumentado la recolección del material no contaminado y que puede ser recuperado y entregado al gestor ambiental de residuos. En el periodo comprendido entre agosto y diciembre del año 2019 se lograron entregar $2316 \mathrm{~kg}$ relativos de material al gestor ambiental de residuos, mientras que en el mismo periodo del año inmediatamente anterior se entregaron $650 \mathrm{~kg}$, representando un aumento cercano al 350\% (figura VII). La principal causa del incremento es que en el año 2018 había una población y consumo similar en la empresa, pero los materiales no eran separados adecuadamente, así que también sufrían contaminación cruzada que imposibilita su fácil recuperación.

Adicionalmente, se ha hecho la compensación del impacto ambiental negativo mediante programas voluntarios de siembra de árboles, así que en el último periodo de han sembrado 35 árboles. Como parte de esta estrategia, la venta de este material permite financiar iniciativas sociales con comunidades vulnerables o en riesgo de exclusión; en el periodo comprendido de agosto a diciembre del año 2019 se lograron recaudar cerca de 300 USD, mientras que en el mismo periodo del año inmediatamente anterior se recaudaron 57 USD aproximadamente (figura VI). 


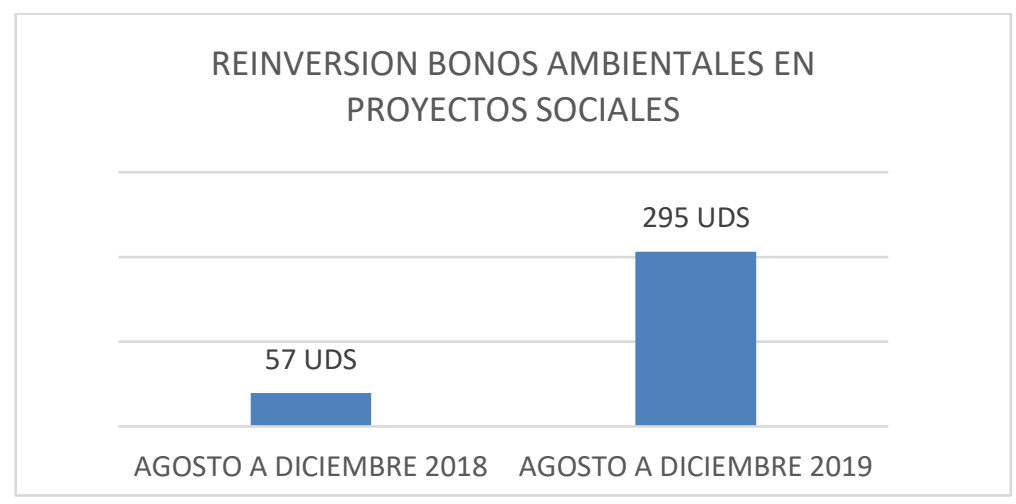

Figura VI.- Incremento de la Reinversión de beneficios económicos del material reciclado.

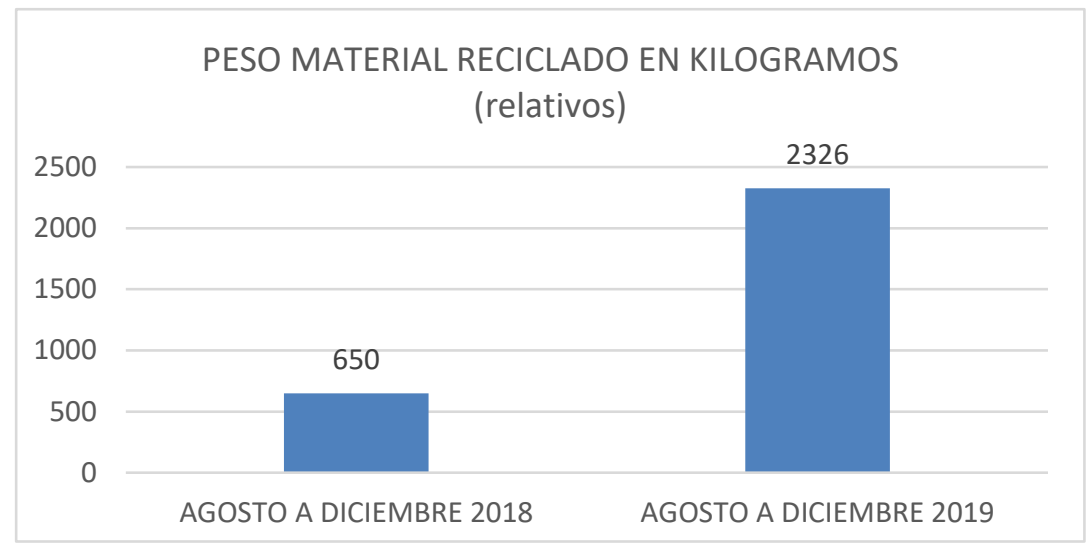

Figura VII.- Incremento del Material reciclado.

5. Concluciones.- El principal objetivo del estudio fue logrado a través de la metodología 4BeGreen, basada en cuatro pasos: 1) Diagnóstico, 2) Caracterización de residuos, 3) Plan de concientización teórica y práctica, 4) Evaluación ecológica y retroalimentación. El diagnóstico está dedicado a entender la situación inicial de la gestión ambiental en la empresa, y el comportamiento de los empleados con herramientas como el "Journey map" para identificar oportunidades de recuperación de materiales, entrenamiento y sensibilización de los empleados. Luego, la caracterización de residuos consiste en identificar los residuos generados en la empresa por actividades de la operación o de backoffice y con esta actividad también se puedo conocer en detalle los errores de separación en los espacios de disposición final de las oficinas. Con la información recopilada, se diseña el plan de concientización teórica y práctica 1) motivar la conciencia e incrementar el conocimiento ambiental, 2) la valorización de los residuos aprovechables y 3) prevención de generación residuos. Finalmente, se ejecuta la evaluación ecológica y retroalimentación para identificar los logros en la separación y valorización de los recursos, así como los desafíos de cara a la transición hacia un sistema circular.

El segundo objetivo del estudio de desarrolló con éxito, ya que se validó la efectividad de la metodología a través de un caso de estudio en una empresa de servicios de exportación y lobby de comercio internacional, ubicada en Colombia. La aplicación de la metodología muestra que la 
capacitación de los empleados y el incremento de su conciencia ambiental permitió la recuperación de $2326 \mathrm{~kg}$ relativos de material reciclable, cuyo beneficio económico valorado en 295 USD se invirtió en proyectos sociales y ambientales. Este resultado prueba que las prácticas ambientales generan ahorros económicos y permiten la reformulación de inversiones. Además, los empleados de la organización se han involucrado en nuevos programas de concienciación ambiental y voluntariado que permiten cumplir con los objetivos de sostenibilidad ambiental y social de la organización. En estas actividades se ha contado con la participación de más de 150 colaboradores, quienes han demostrado la implementación de prácticas de consumo sostenible y especialmente en la adecuada separación de residuos.

A nivel práctico se espera que en el largo plazo los empleados de la organización autogestionen el programa para mantener y mejorar la correcta separación de residuos, el uso de los puntos ecológicos, la eliminación de canecas personales, la creación de nuevos hábitos, concientización sobre consumo diario, incluso en los hogares. Así mismo, el sentido de retribución de los colaboradores los ha hecho sentir más a gusto en su trabajo, empatía, y satisfacción.

Sin embargo, aún permanecen barreras como la disponibilidad de tiempo para participar en las actividades de aprendizaje y gestión, una minoría de los colaboradores aun no muestra interés en el proceso, el proyecto ha sido o cuenta con un presupuesto propio y la pandemia del COVID19 ha interrumpido algunas de las actividades de concientización en las instalaciones de la organización lo cual impide la trazabilidad de los residuos de los colaboradores que trabajan desde sus hogares.

Finalmente, el estudio cuenta con algunas limitaciones. La primera de ellas es que la metodología 4BeGreen estás especialmente diseñada para disminuir los residuos que generan los empleados directamente, mientras que residuos como los eléctricos o electrónicos no han sido incluido. En estudios futuros hay una gran oportunidad para identificar estrategias que permitan ampliar el tipo de materiales que se pueden recuperar en empresas de servicios.

6. Agradecimientos.- Esta investigación es fruto del apoyo del "Programa ViVerde - Vive La Sostenibilidad" y proyecto "Desafíos y Oportunidades de la Implementación de la Economía Circular en las Empresas” ID00009299 de la Pontificia Universidad Javeriana. 


\section{Referencias.-}

[1] UN Global Compact, "La misión del Pacto Mundial: 10 Principios + 17 ODS," Pacto Mundial ONU, 2017. [Online]. Available: http://www.pactomundial.org/2017/03/10principios-17-ods/. [Accessed: 10-Aug-2020].

[2] Prieto-Sandoval, V. et al., "Towards a consensus on the circular economy," J. Clean. Prod., vol. 179, no. April, pp. 605-615, 2018.

[3] Park, J. et al., "Creating integrated business and environmental value within the context of China's circular economy and ecological modernization," J. Clean. Prod., vol. 18, no. 15, pp. 1492-1499, Oct. 2010.

[4] Prieto-Sandoval, V. et al., "Economía circular: Relación con la evolución del concepto de sostenibilidad y estrategias para su implementación [Circular economy: Relationship with the evolution of the concept of sustainability and strategies for its implementation]," Mem. Investig. en Ing., no. 15, p. 85, 2017.

[5] Bakker, C. et al., "Products that go round: exploring product life extension through design," J. Clean. Prod., vol. 69, pp. 10-16, 2014.

[6] European Commission, "Closing the loop - An EU action plan for the Circular Economy," Brussels, 2015.

[7] Moigne, R. Le, "Why Reverse Logistics Is an Essential Part of a Circular Economy," Circulate News- Ellen MacArthur Foundation, 2016.

[8] Franco, M. A., "Circular economy at the micro level: A dynamic view of incumbents' struggles and challenges in the textile industry," J. Clean. Prod., vol. 168, pp. 833-845, 2017.

[9] Ormazabal, M. et al., "Environmental training at companies. The case of Volkswagen Navarra," in 2 nd International Conference on Higher Education Advances, 2017, pp. 1077-1084.

[10] Matus, K. J. M. et al., "Green chemistry and green engineering in China: drivers, policies and barriers to innovation,” J. Clean. Prod., vol. 32, pp. 193-203, Sep. 2012.

[11] Ormazabal, M. et al., "An Overview of the Circular Economy Among SMEs in the Basque Country: A Multiple Case Study,” J. Ind. Eng. Manag., vol. 9, no. 5, pp. 1047-1058, Dec. 2016.

[12] ISO, "Environmental management - The ISO 14000 family of International Standards." ISO Central Secretariat 1, Geneve, Switzerland, 2009.

[13] Prieto-Sandoval, V. et al., "ECO-labels as a multidimensional research topic: Trends and opportunities," J. Clean. Prod., vol. 135, pp. 806-818, 2016.

[14] Thøgersen, J. et al., "Consumer decision making regarding a 'green' everyday product," Psychol. Mark., vol. 29, no. 4, pp. 187-197, 2012.

[15] Smith, R., "Waste management," in Salem Press Encyclopedia, 2018.

[16] Cramer, J., "Environmental management: from 'fit'to 'stretch,"” Bus. Strateg. Environ., vol. 7, no. 3, pp. 162-172, 1998.

[17] Ormazabal, M. et al., "Evolutionary Pathways of Environmental Management in UK 
Companies," Corp. Soc. Responsib. Environ. Manag., vol. 22, no. 3, pp. 169-181, May 2015.

[18] ONU Habitat, "Campaña global \#WasteWiseCities," ONU Habitat, 2019. [Online]. Available: $\quad$ https://www.onuhabitat.org.mx/index.php/campana-globalwastewisecities\#: :text=ONU-Habitat alienta a todas,antes y después del consumo. [Accessed: 10-Aug-2020].

[19] Hatch, Mary Jo; Cunliffe, A. L., Organization Theory: Modern, Symbolic, and Postmodern Perspectives, 3rd ed. New York, NY, USA: Oxford University Press, 2013.

[20] Torres, S. and A. Mejía-Villa, “A contemporary view of the concept of administration," Cuad. Adm., vol. 19, no. 32, pp. 111-133, 2006.

[21] Morgan, G., Images of Organization, 2nd ed. Newbury Park, CA.: Sage Publications, 1997.

[22] Veleva, V. et al., "The need for better measurement and employee engagement to advance a circular economy: Lessons from Biogen's 'zero waste' journey," J. Clean. Prod., vol. 154, pp. 517-529, 2017.

[23] Cohen-Rosenthal, E., "A walk on the human side of industrial ecology," J. Compos. Mater., vol. 33, no. 2, pp. 928-940, Oct. 2000.

[24] Chan, E. S. W. et al., "What drives employees' intentions to implement green practices in hotels? The role of knowledge, awareness, concern and ecological behaviour," Int. J. Hosp. Manag., vol. 40, pp. 20-28, 2014.

[25] WTO, "La importancia del comercio de servicios," in EL FUTURO DEL COMERCIO DE SERVICIOS, WTO, 2019, pp. 56-91.

[26] Residuos, M. and M. V. Pl, "Cuentas medioambientales: Cuentas de los residuos 2017," Inst. Nac. Estad., vol. 2017, pp. 1-7, 2019.

[27] Cardona Gallo, M., "Minimización de Residuos: una política de gestión ambiental empresarial," Prod. + Limpia, vol. 1, no. 2, p. Julio-Diciembre 2006, 2006.

[28] Zainal, Z., "Case study as a research method,” J. Kemanus., vol. 9, no. Journal Article, pp. $1-6,2007$.

[29] Sánchez-Muñoz, M. del P. et al., "Gestión de residuos sólidos urbanos en América Latina: un análisis desde la perspectiva de la generación," Rev. Finanz. y Polit. Econ., vol. 11, no. 2, pp. 321-336, 2019.

[30] Calderón Márquez, A. J. and E. W. Rutkowski, "Waste management drivers towards a circular economy in the global south - The Colombian case," Waste Manag., vol. 110, pp. 53-65, 2020.

[31] Sánchez-Muñoz, M. del P. et al., “Análisis de la opinión de los hogares sobre la gestión de los residuos sólidos domiciliarios en Bogotá,” Semest. Económico, vol. 22, no. 52, pp. 97129, 2019.

[32] Jaca, C. et al., Economía circular - Guía para Pymes. Pamplona, España.: EUNSA, 2018.

[33] Kumar, V., 101 design methods: A structured approach for driving innovation in your organization. John Wiley \& Sons, 2012. 